\title{
Antiinflammatory profiles during primary SIV infection in African green monkeys are associated with protection against AIDS
}

\author{
Christopher Kornfeld, ${ }^{1}$ Mickaël J.-Y. Ploquin, ${ }^{1}$ Ivona Pandrea, ${ }^{2}$ Abdourahmane Faye, ${ }^{3}$ \\ Richard Onanga, ${ }^{2}$ Cristian Apetrei, ${ }^{2}$ Virginie Poaty-Mavoungou, ${ }^{2}$ Pierre Rouquet, ${ }^{2}$ \\ Jérôme Estaquier, ${ }^{4}$ Lorenzo Mortara, ${ }^{1}$ Jean-François Desoutter, ${ }^{5}$ Cécile Butor, ${ }^{5,6}$ \\ Roger Le Grand,7 Pierre Roques, ${ }^{2,7}$ François Simon, ${ }^{2,3}$ Françoise Barré-Sinoussi, ${ }^{1}$ \\ Ousmane M. Diop, ${ }^{3}$ and Michaela C. Müller-Trutwin ${ }^{1}$
}

\footnotetext{
${ }^{1}$ Unité de Biologie des Rétrovirus, Institut Pasteur, Paris, France. ${ }^{2}$ Centre International de Recherche Médicale, Franceville, Gabon. ${ }^{3}$ Laboratoire de Rétrovirologie, Institut Pasteur, Dakar, Senegal. ${ }^{4}$ Unité de Recherche et d'Expertise Physiopathologie des Infections Lentivirales, Institut Pasteur, Paris, France. 5INSERM 567, UMR CNRS 8104, IFR 116, Département d'Immunologie, Institut Cochin, Paris, France. ${ }^{6}$ Université Paris VII Denis Diderot, Paris, France. ${ }^{7}$ Commissariat à l'Energie Atomique, Fontenay aux Roses, France.
}

\begin{abstract}
T cell activation levels in HIV infection are predictive of AIDS progression. We searched for the immunological correlates of protection against disease progression by studying the early stages of nonpathogenic SIV infection in African green monkeys (SIVagm). The African green monkeys (AGMs) displayed high peak viremias and a transient decline in levels of blood $\mathrm{CD4}^{+}$and $\mathrm{CD8}^{+} \mathrm{T}$ cells between days 5 and 17 after infection. A concomitant increase in levels of $\mathrm{CD}^{+} \mathrm{DR}^{+}, \mathrm{CD8}^{+} \mathrm{DR}^{+}$, and $\mathrm{CD8}^{+} \mathrm{CD}^{2} 8^{-}$cells was detected. After the third week, $\mathrm{T}$ cell activation returned to baseline levels, which suggested a protective downregulation of $T$ cell activation. A very early ( 24 hours after infection) and strong induction of TGF- $\beta 1$ and FoxP3 expression was detected and correlated with increases in levels of $\mathrm{CD}^{+} \mathrm{CD}^{+} 5^{+}$and $\mathrm{CD8}^{+} \mathrm{CD}^{-} 5^{+} \mathrm{T}$ cells. This was followed by a significant increase in levels of IL-10, whereas IFN- $\gamma$ gene upregulation was more transient, and levels of TNF- $\alpha$ and $M I P-1 \alpha / \beta$ transcripts did not increase in either blood or tissues. The profiles were significantly different during primary SIV infection in macaques (SIVmac); that is, there was a delayed increase in IL-10 levels accompanied by moderate and persistent increases in TGF- $\beta$ levels. Together, our data show that SIVagm infection is associated with an immediate antiinflammatory environment and suggest that TGF- $\beta$ may participate in the generation of Tregs, which may prevent an aberrant chronic $T$ cell hyperactivation.
\end{abstract}

\section{Introduction}

HIV-1 infection is characterized by chronic T cell hyperactivation. Increased $\mathrm{T}$ cell activation from the initial stage of infection has predictive value for AIDS progression even before seroconversion and independently of viral load (VL) (1-4). The precise mechanisms leading to this aberrant chronic $\mathrm{T}$ cell activation remain unclear. The study of SIV infections in nonhuman primate models has contributed to an understanding of the mechanisms underlying $\mathrm{CD}^{+}$ $T$ cell depletion and AIDS. The animal model that best reflects HIV infection in humans is SIV infection in macaques (SIVmac). SIV infections are generally only nonpathogenic in African nonhuman primate species, the natural hosts of SIV, such as African green monkeys (AGMs), mandrills (MNDs), sooty mangabeys (SMs), and chimpanzees (CPZs) (5). During SIVagm, SIVsm, SIVmnd-1, and SIVcpz infections in their respective hosts, VLs have been shown to be similar to those recorded for pathogenic HIV-1 and SIVmac infections (6). Furthermore, SIVagm replicates in lymphoid tissues, including the gut $(6,7)$. Thus, these studies have shown that viral replication alone is not sufficient to drive $\mathrm{CD}^{+} \mathrm{T}$ cell decline.

Nonstandard abbreviations used: AGM, African green monkey; BAL, bronchoalveolar lavage; BALC, BAL cell; LNLC, LN lymphocyte; SIVagm, SIV infection in African green monkeys; SM, sooty mangabey; VL, viral load.

Conflict of interest: The authors have declared that no conflict of interest exists.

Citation for this article: J. Clin. Invest. 115:1082-1091 (2005).

doi:10.1172/JCI200523006
Despite high levels of VLs, natural hosts show distinct lymphocyte activation profiles during chronic infection compared with humans and macaques. Naturally infected AGMs and SMs do not exhibit significant LN follicular hyperplasia $(8,9)$ or infiltration of $\mathrm{CD}^{+}$cells into LN germinal centers (9). Naturally infected MNDs and SMs exhibit nearly unchanged percentages of $\mathrm{CD} 4^{+} \mathrm{DR}^{+} \mathrm{T}$ cells and only slight increase in the percentages of $\mathrm{CD}^{+} \mathrm{DR}^{+} \mathrm{T}$ cells in blood $(10,11)$. Long-term SIVsm infection in SMs is characterized by normal $\mathrm{T}$ cell turnover and decreased IFN- $\gamma$ expression that correlates with low CTL activity $(8,11,12)$. Finally, chronically infected AGMs, SMs, and CPZs failed to show enhanced peripheral $\mathrm{CD}^{+} \mathrm{T}$ cell apoptosis levels $(11,13)$. Data on $\mathrm{T}$ cell activation and cytokine profiles during the initial stage of SIVagm in AGMs are crucially needed in order to understand the very early events that will shape immune responses and prevent the aberrant chronic $\mathrm{T}$ cell activation despite continuous viral replication and the following questions must be addressed: (a) Are the T cells activated during primary infection in response to SIVagm? (b) Are the cytokine profiles pro- or antiinflammatory? (c) Is the low activation associated with tolerance toward SIVagm?

To address these questions, we studied $T$ cell activation patterns in distinct tissues of SIVagm-infected AGMs during primary infection. $\mathrm{CD}^{+}$and $\mathrm{CD}^{+} \mathrm{T}$ cell subpopulations were examined in peripheral blood as well as in secondary lymphoid organs. To analyze the early orientation of the immune response, we compared the cytokine profiles of 3 distinct compartments - blood 

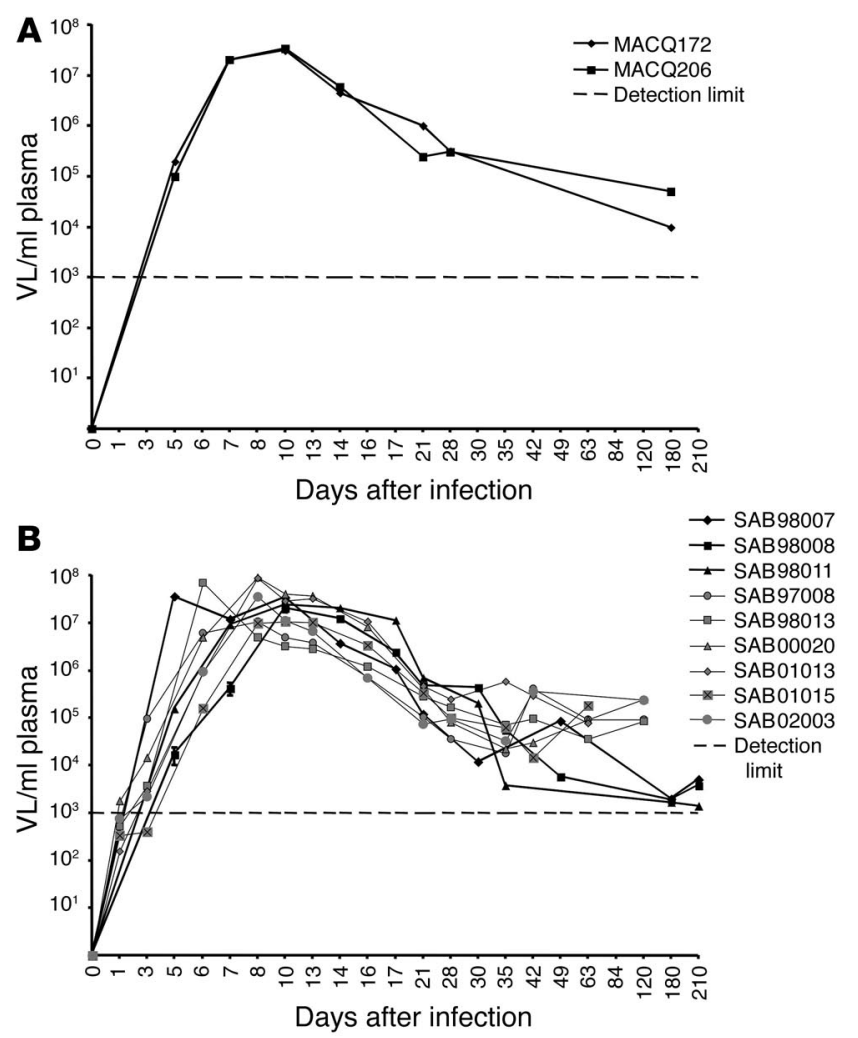

Figure 1

Plasma viral RNA copy numbers were measured by real-time PCR (cut off value, $10^{3}$ copies $/ \mathrm{ml}$ of plasma). (A) Macaques infected with SIVmac251. (B) AGMs infected with SIVagm.sab92018.

(i.e., at a systemic level), LN, and peripheral tissue (by bronchoalveolar lavage $[\mathrm{BAL}]$ - at initiation and effector sites of the immune response. This study reveals that SIVagm infection in AGMs can lead to a transient $T$ cell activation during primary infection but that AGMs mount an immediate antiinflammatory cytokine environment. This raises the possibility of a Treg induction, which may then control $\mathrm{T}$ cell turnover rates.

\section{Results}

Transient decline in blood $\mathrm{CD} 4^{+} \mathrm{T}$ cells during primary SIVagm infection. Nine AGMs infected with SIVagm.sab92018 as well as 2 control animals were included in this study. To allow comparison between pathogenic SIVmac and nonpathogenic SIVagm infection under the same experimental conditions, we included 2 SIVmac251infected macaques as well as 2 control macaques as internal control for phenotypic analysis of $\mathrm{CD}^{+}$and $\mathrm{CD}^{+} \mathrm{T}$ cells (Figures 1-3). Both infected macaques progressed rapidly toward disease, developing AIDS within 7 months after infection. Plasma VLs were very similar in SIVmac-infected macaques and SIVagm-infected AGMs (Figure 1). VLs peaked between 6 and 10 days after infection $\left(3 \times 10^{7}\right.$ to $8 \times 10^{7}$ copies $/ \mathrm{ml}$ of plasma). After day 35 , the plasma VLs ranged from of $2 \times 10^{3}$ to $8 \times 10^{4}$ copies $/ \mathrm{ml}$ and were within the range previously recorded by us and others in chronically infected AGMs and macaques $(6,14,15)$.

We then compared the early $\mathrm{T}$ cell dynamics in macaques and AGMs. In both SIVmac-infected macaques (MACQ172 and MACQ205), the blood $\mathrm{CD}^{+} / \mathrm{CD}^{+} \mathrm{T}$ cell ratios decreased starting from day 10 after infection. (Figure 2A). After day 10, the absolute number $\mathrm{CD} 4^{+} \mathrm{T}$ cells remained low compared with baseline, whereas numbers of $\mathrm{CD}^{+} \mathrm{T}$ cells returned to baseline (data not shown). In AGMs, an early significant decline was also detected, reaching a maximum between days $7(P<0.0001)$ and 10 $(P<0.0001)$ (Figure 2B). This decline was due to a more pronounced decrease in the absolute number of $\mathrm{CD}^{+} \mathrm{T}$ cells compared with $\mathrm{CD}^{+} \mathrm{T}$ cells (data not shown). Both $\mathrm{CD} 4^{+}$and $\mathrm{CD} 8^{+} \mathrm{T}$ cell counts reached preinfection values after day 10 and remained stable. There was a negative correlation between plasma SIVagm RNA levels and $\mathrm{CD}^{+} \mathrm{T}$ cell counts $(\rho=-0.541 ; P<0.0001)$ during primary SIVagm infection. Together, our results indicate that primary SIVagm infection in AGMs, like pathogenic SIVmac infections in macaques, can lead to a significant decrease in the absolute number of $\mathrm{CD}^{+} \mathrm{T}$ cells in blood.

To determine whether modulations in blood $\mathrm{T}$ cell subsets are reflected in secondary lymphoid tissues, we analyzed $\mathrm{CD}^{+}$ and $\mathrm{CD}^{+} \mathrm{T}$ cell percentages in $\mathrm{LN}$ during SIVagm and SIVmac infections. Whereas the 2 mock-infected control macaques had a stable $\mathrm{CD}^{+} / \mathrm{CD}^{+} \mathrm{T}$ cell ratio in $\mathrm{LN}$ throughout the experimental follow-up, both SIVmac-infected macaques exhibited a decrease during the acute phase of the infection. This decline was more pronounced during the chronic phase of infection (Figure 2C). By contrast, SIVagm-infected AGMs showed no significant changes in $\mathrm{CD}^{+} / \mathrm{CD}^{+} \mathrm{T}$ cell ratios within $\mathrm{LN}$ at any time during the period of observation (Figure 2D). This observation is consistent with our previous finding of a lack of $\mathrm{CD} 8^{+} \mathrm{T}$ cell infiltrations into LN germinal centers (9).

Transient increases of activated $\mathrm{CD} 4^{+}$and $C D 8^{+} T$ cells in blood during primary SIVagm infection. To determine whether decreases in circulating $\mathrm{CD}^{+}$and $\mathrm{CD}^{+} \mathrm{T}$ cells in AGMs were associated with changes in $\mathrm{T}$ cell activation levels, we performed a comparative analysis of $\mathrm{T}$ cell activation markers in blood and LN cells during primary SIVagm and SIVmac infection. We focused our comparative analysis on 2 cell-surface markers (DR and CD28), whose expression levels correlate with disease progression in HIV-1/SIVmac infections $(1,3,16)$.

We analyzed $\mathrm{T}$ cell activation in macaques and AGMs. None of the SIVmac-infected macaques studied showed significant changes in the proportion of circulating DR-expressing $\mathrm{CD} 4^{+} \mathrm{T}$ cells during primary infection, but the proportion was enhanced after day 28 (Figure 3A). By contrast, in AGMs, there was a significant increase in the percentages of $\mathrm{CD} 4^{+} \mathrm{DR}^{+}$cells only at early time points, i.e. at days $7(P=0.003)$ and $10(P<0.0001)$ (Figure $3 \mathrm{~B})$. Activation profiles within the circulating $C D 8^{+} \mathrm{T}$ cell population mirrored those observed for circulating CD4 ${ }^{+} \mathrm{T}$ cells. During primary SIVmac infection in macaques, an early, slight increase in $\mathrm{CD}^{+} \mathrm{DR}^{+} \mathrm{T}$ cells was followed by a persistent increase during chronic infection (Figure 3C) concomitantly to a continuous decrease of $\mathrm{CD} 8^{+} \mathrm{CD} 28^{+} \mathrm{T}$ cells (data not shown). In AGMs, the baseline percentage of $\mathrm{DR}^{+}$ peripheral blood $\mathrm{CD}^{+} \mathrm{T}$ cells was more variable than that seen in macaques (ranging from $8 \%$ to $62 \%$ ). After SIVagm infection, the percentages of $\mathrm{CD}^{+} \mathrm{DR}^{+} \mathrm{T}$ cells were significantly increased between days 8 and 21 ( $P \leq 0.044$ for each) (Figure 3D). Values then declined to baseline levels by day 28. AGMs also exhibited significant decreases in $\mathrm{CD} 8{ }^{+} \mathrm{CD} 28^{+} \mathrm{T}$ cells between days 5 and 21 $(P \leq 0.035$ for each) (data not shown). After day 21, the percentages of $\mathrm{CD} 8^{+} \mathrm{CD} 28^{+} \mathrm{T}$ cells returned to their preinfection values. We thus confirm a lack of increased $T$ cell activation during the chronic phase of infection and reveal for the first time to our knowledge that $\mathrm{T}$ cell activation can occur during primary SIVagm infection. 
A

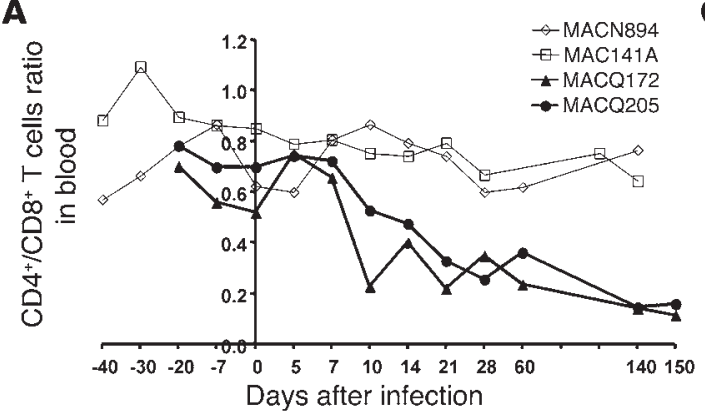

B

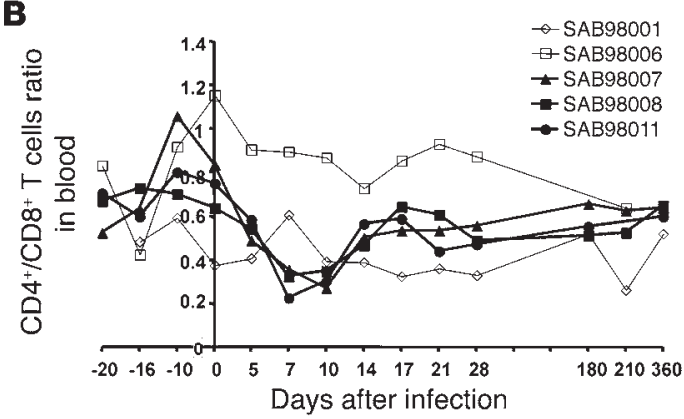

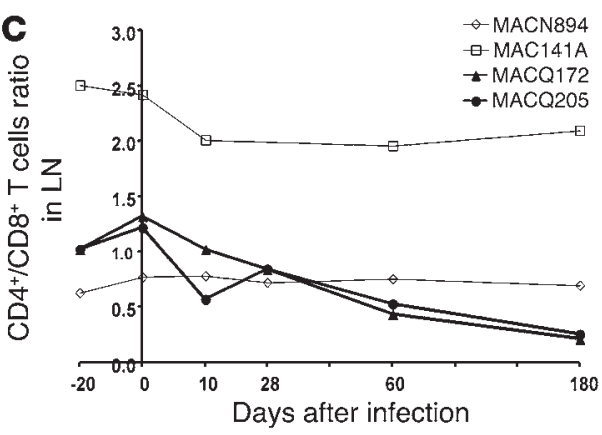

D

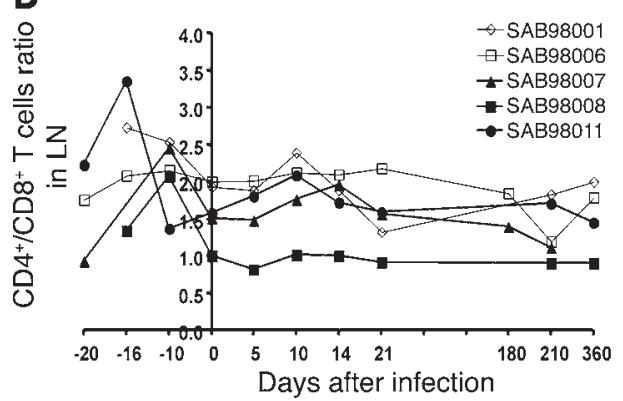

Figure 2

$\mathrm{CD}^{+} / \mathrm{CD}^{+} \mathrm{T}$ cell ratio during SIV infection. Data obtained with macaques ( $\mathbf{A}$ and $\mathbf{C})$ and AGMs (B and D). CD4+/CD8 ${ }^{+}$ $T$ cell ratios during SIV infection were determined in blood (A and $\mathbf{B}$ ) and in LN (C and $\mathbf{D}$ ) for 3 SIVagm-infected AGMs (SAB98007, SAB98008, and SAB98011), 2 SIVmac251-infected macaques (MACQ172 and MACQ205), and mock-infected controls (SAB98001, SAB98006, MACN894, and MAC141A). Similar profiles to these shown here were obtained in blood of the 6 other AGMs (SAB97008, SAB98013, SAB00020, SAB01013, SAB01015, and SAB02003) (data not shown). Open and filled symbols represent uninfected and SIV-infected monkeys, respectively.
The percentages of activated $C D 8^{+}$T cells in AGM LNs remain unaltered after SIVagm infection. There was no significant change in the frequency of $\mathrm{LN} \mathrm{CD} 4{ }^{+} \mathrm{DR}^{+}$and $\mathrm{CD} 4^{+} \mathrm{CD} 28^{+} \mathrm{T}$ cells from macaques and AGMs after infection (data not shown). An increase in $\mathrm{CD}^{+}{ }^{+} \mathrm{DR}^{+}$and $\mathrm{CD} 8^{+} \mathrm{CD} 28^{-} \mathrm{T}$ cells was observed after 60 days in the SIVmac-infected macaques, whereas in AGMs, no significant changes were evidenced in LN (data not shown). During the chronic phase of infection, there is therefore, as expected, a lower level of $\mathrm{CD}^{+} \mathrm{T}$ cell activation in the $\mathrm{LN}$ of AGMs compared with macaques. That the frequencies of DRand CD28-positive $\mathrm{CD}^{+} \mathrm{T}$ cells in $\mathrm{LN}$ were unaltered during primary infection contrasts with the changes detected in the
A

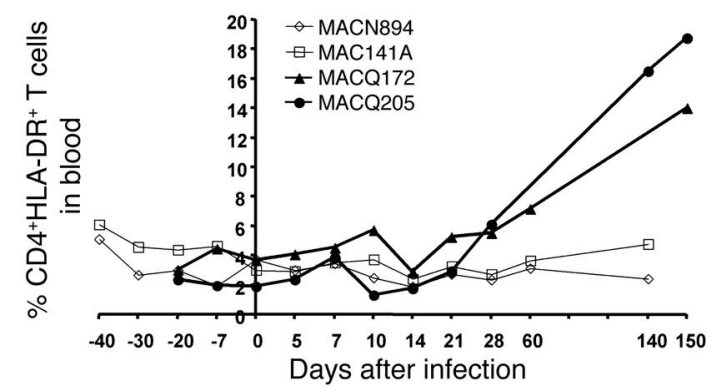

B

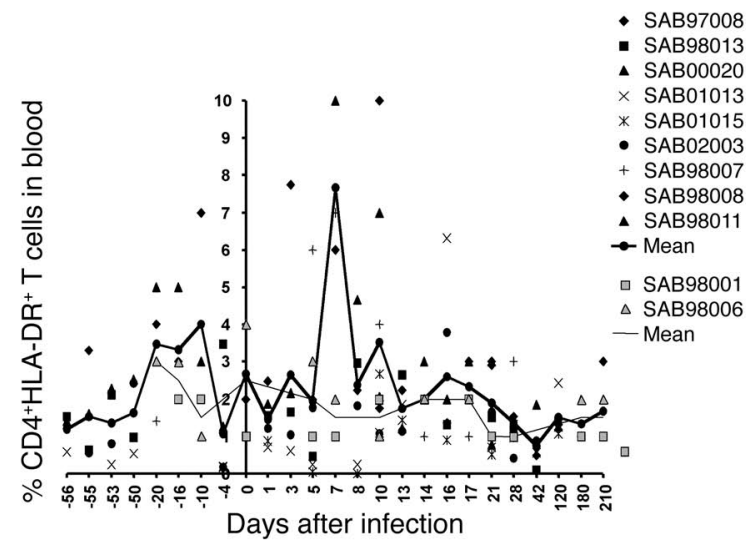

C

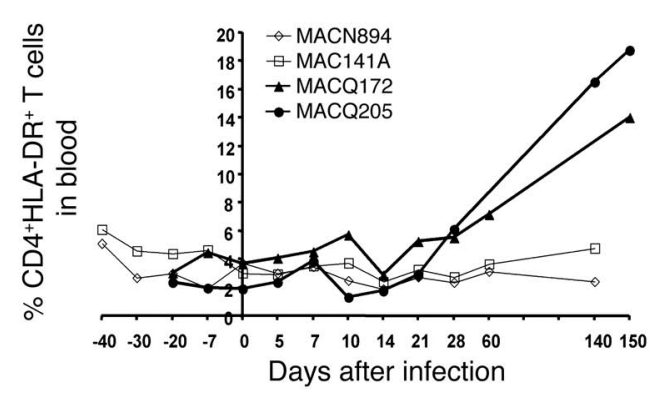

D

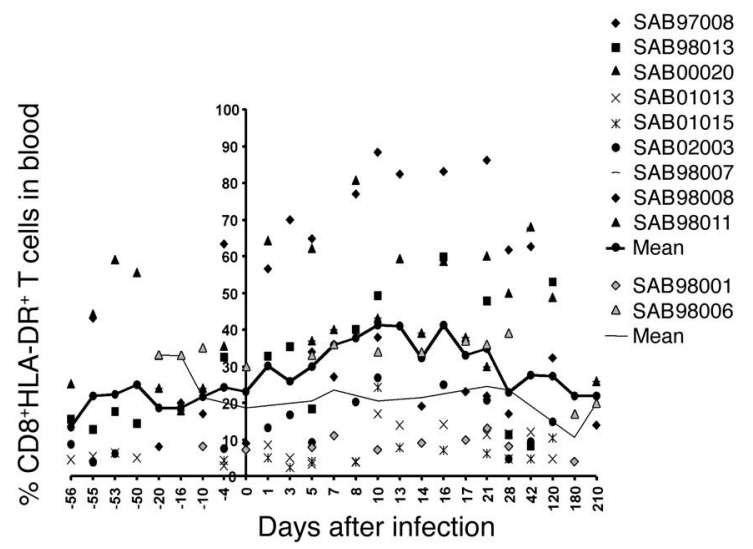

Figure 3

Frequency of circulating activated T cells during SIV infection. Percentage of HLA-DR+ cells within blood CD4+ $\mathrm{T}(\mathbf{A}$ and $\mathbf{B})$ and CD8+ $\mathrm{T}$ cells $(\mathbf{C}$ and D). (A and C) SIVmac-infected macaques. Open and filled symbols represent uninfected and SIV-infected macaques, respectively. (B and D) Bold and thin lines represent the mean from 9 SIVagm-infected AGMs and 2 uninfected AGMs (SAB98001 and SAB98006), respectively. 
A
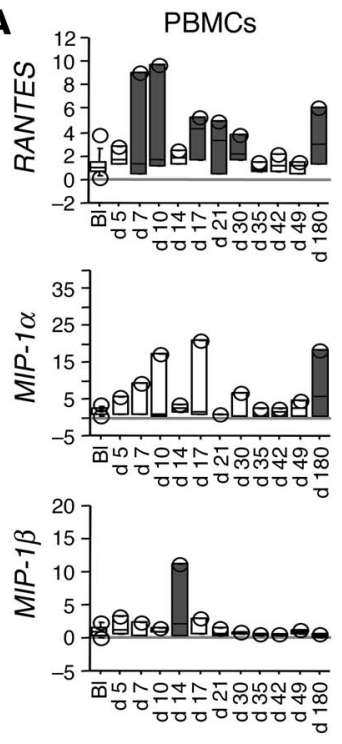

B
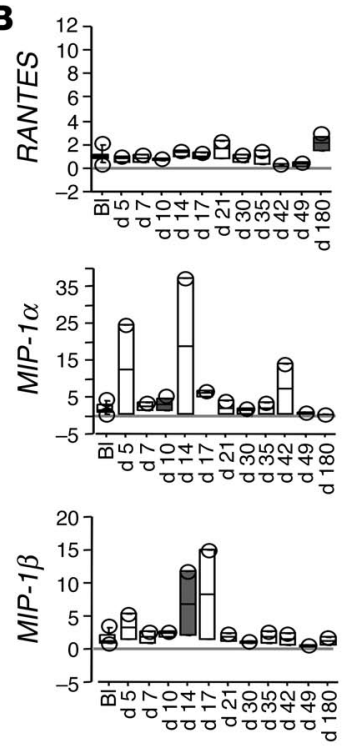
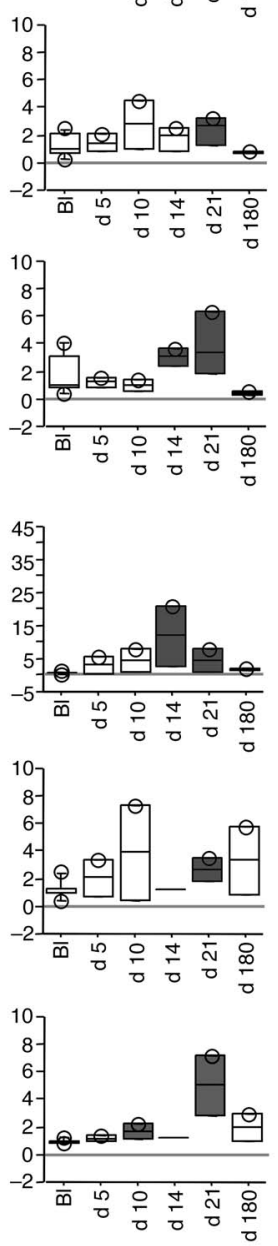

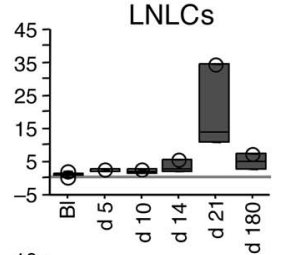

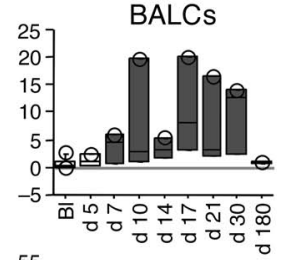
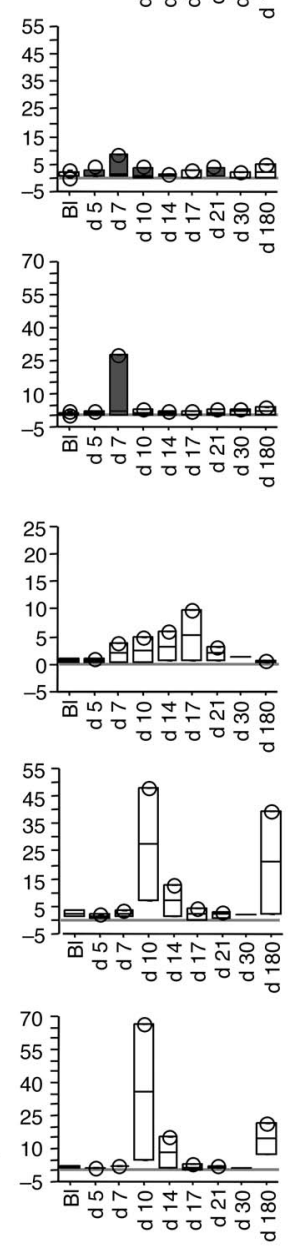

\section{Figure 4}

Expression of $\beta$ chemokine gene transcripts in blood and tissues of 3 SIVagm-infected AGMs (SAB98007, SAB98008, and SAB98011) (A) and 2 mock-infected AGMs (SAB98001 and SAB98006) (B). Values at time points after SIV inoculation (days 5-180) are expressed as $n$-fold increases relative to values found before infection (BI). Gene expressions levels are represented by box plots. Gray boxes indicate statistically significant increases $(P<0.05$, Wilcoxon signed rank test) relative to baseline.

in both SIV- and mock-infected animals and were thus not considered to be specifically induced by SIVagm infection (Figure 4, A and B). Changes in the expression levels of $M I P-1 \alpha$ and $M I P-1 \beta$ genes were less frequently observed in infected animals than for RANTES and/or also observed in the mock-infected AGMs (Figure 4, A and B) after infection and throughout acute infection $(P \leq 0.027$ for each). In summary, these observations demonstrate that SIVagm infection in AGMs leads to induction of RANTES, CCR1, and CCR5 gene expression in PBMCs and BALCs, whereas no infection-specific increases were detected in LN. Furthermore, no or only very modest gene expression changes of $M I P-1 \alpha$ and $M I P-1 \beta$ were detected.

Increased IL-10/TNF-a ratios during primary SIVagm infection. To further explore the possibility that proinflammatory cytokine expression is dampened during SIVagm infection, we analyzed the gene expression levels of TNF- $\alpha$ and $I L-10$. Their expression was first measured in the 3 tissue compartments of the same 5 AGMs analyzed for chemokine gene expression (Figure 5), as well as in the 6 other AGMs at earlier time points with shorter intervals in PBMCs (Figure 6). TNF- $\alpha$ gene expression was increased in PBMCs at days 5, 10 , and 17 after infection $(P<0.042$ for each) and in LN at days $5(P=0.008)$ and $21(P=0.007)$, but mock-infected control AGMs also exhibited similar TNF- $\alpha$ gene expression changes in both PBMCs and LN (Figure 5). The increases in TNF- $\alpha$ expression therefore appear not to be SIV specific but rather due to other factors, such as repeated sampling and/or injection of plasma. In BALCs of both SIVagm- and mock-infected AGMs, no significant changes in TNF- $\alpha$ periphery but is consistent with the absence of $\mathrm{CD} 4^{+} / \mathrm{CD}^{+} \mathrm{T}$ cell ratio changes.

Expression of RANTES, CCR1, and CCR5 transcripts is transiently upregulated in acutely SIVagm-infected AGMs. The presence of higher levels of activated $T$ cells and of infiltrating cells in $L N$ in pathogenic infections may result from a heightened state of inflammation. We searched for differences in the early pro- and antiinflammatory cytokine profiles induced during primary infection. We analyzed the profiles in AGMs in 3 distinct compartments: PBMCs, LN lymphocytes (LNLCs), and BAL cells (BALCs), a peripheral compartment that has been found to present signs of inflammation in acutely SIVmac-infected macaques $(17,18)$. The 3 compartments were studied in 5 AGMs ( 3 randomly selected SIVagm-infected AGMs and the 2 mock-infected control AGMs). We first measured $\beta$ chemokine expression as a marker for inflammation.

During primary SIVagm infection, RANTES gene expression was significantly increased (at days 7, 10, 17, 21, and 30 in PBMCs $(P \leq 0.04$ for each) and in BALCs from days 7 to $30(P \leq 0.027$ for each). In LN, increases in RANTES expression levels were observed gene expression were detected at any time during the study period (Figure 5B), in contrast to what has been observed in macaques (19). The expression of the $I L-10$ gene in PBMCs and BALCs from SIVagm-infected AGMs (Figure 5A), but not mock-infected controls (Figure 5B), was significantly increased starting from day 7 until day 30 after infection $(P<0.05$ for each). In $L N, I L-10$ gene expression was significantly increased at days $14(P=0.043)$ day 21 $(P=0.008)$ in infected AGMs and significantly increased at day 14 $(P=0.043)$ in mock-infected animals.

In summary, the above studies indicated TNF- $\alpha /$ IL-10 ratios in favor of IL-10, specially in PBMCs and BALCs. We extended our analysis of TNF- $\alpha$ and $I L-10$ gene expression in PBMCs to the 6 other AGMs. The kinetics of sampling were similar to those carried out above, except that we also collected blood at earlier time points (days 1 and 3 after infection), since the inflammatory balance might be established very early. The $6 \mathrm{AGMs}$ also exhibited the same TNF- $\alpha /$ IL-10 ratio in favor of IL-10 in PMBC (Figure 6A).

To further confirm this antiinflammatory cytokine environment in response to SIVagm infection, we measured TNF- $\alpha$ and IL-10 
A
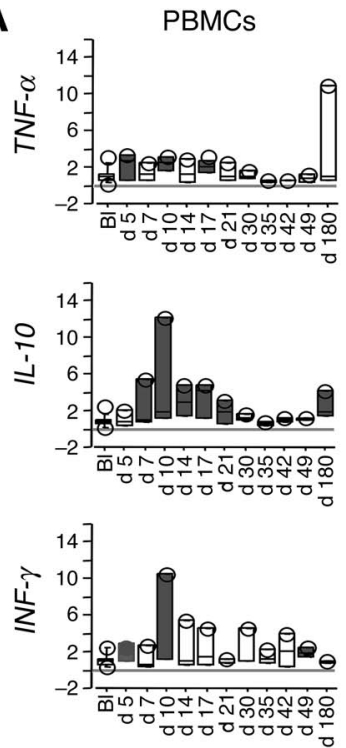

B
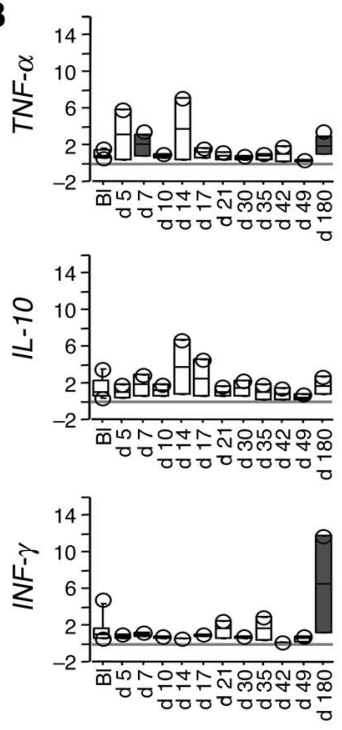
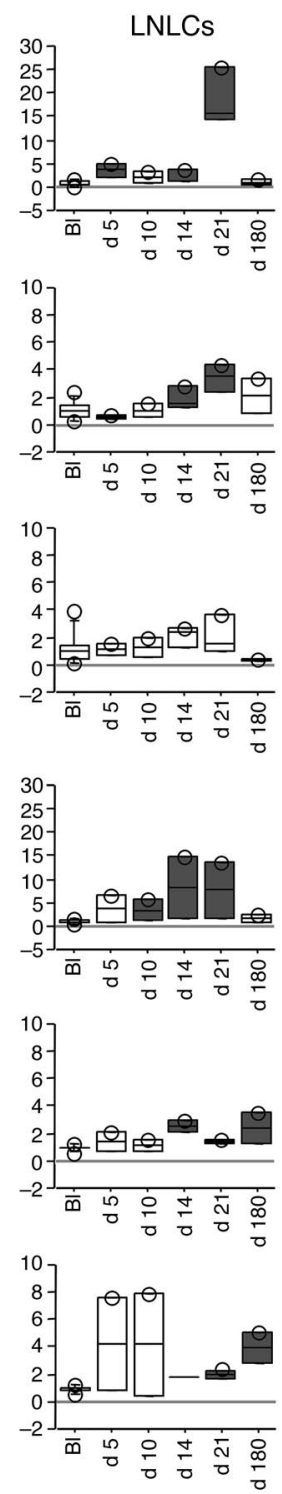
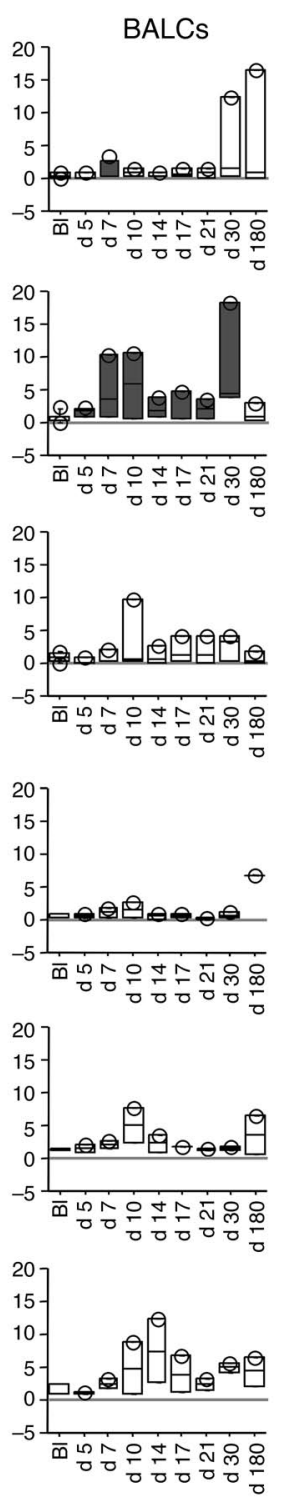

Figure 5

$I L-10, T N F-\alpha$, and IFN- $\gamma$ gene expressions in PBMCs, LNLC, and BALCs of 3 SIVagm-infected AGMs (SAB98007, SAB98008, and SAB98011) (A) and 2 mock-infected AGMs (SAB98001 and SAB98006) (B). Gray boxes indicate statistically significant increases $(P<0.05$, Wilcoxon signed rank test) as compared with preinfection baseline values. See also legend to Figure 4.

SIVagm-infected and 2 mock-infected AGMs. In LNLCs and BALCs, no statistically significant changes in $I F N-\gamma$ gene expression were detected at any time after SIVagm infection (Figure 5). In PBMCs, the SIVagm-infected animals demonstrated a significant increase at days $5(P=0.018)$ and 10 $(P=0.033)$. In the 6 other AGMs, we also observed a similar transient increase in PBMCs at days $10(P=0.005)$ and 16 $(P=0.019)$ (Figure 6A). To summarize, the analysis of $I F N-\gamma$ gene expression detected increases only in PBMCs, but not in LNLCs and BALCs, in contrast to IL-10. In PBMCs, the increases in IFN- $\gamma$ gene expression appeared to be more transient than those in $I L-10$ expression.

Immediate increases of $\mathrm{CD}^{+} \mathrm{CD} 25^{+}$and $\mathrm{CD} 8^{+} \mathrm{CD} 25^{+} \mathrm{T}$ cells in blood. The above data reveal that primary SIVagm infection in AGMs is associated with a transient activation of $\mathrm{T}$ cells, TNF- $\alpha / \mathrm{IL}-10$ ratios in favor of IL-10, and a weak $I F N-\gamma$ response. These observations raised the hypothesis that Tregs are induced after SIVagm infection of AGMs. To evaluate this possibility more closely, we analyzed the frequency of $\mathrm{CD}^{+} \mathrm{CD} 25^{+}$and $\mathrm{CD} 8{ }^{+} \mathrm{CD} 25^{+} \mathrm{T}$ cells in $6 \mathrm{AGMs}$ from time points directly after infection (day 1 ) through day 430 after infection.

At days 1 and 6 , percentages of $\mathrm{CD}^{+} \mathrm{CD} 25^{+}$and $\mathrm{CD}^{+} \mathrm{CD} 25^{+} \mathrm{T}$ cells in peripheral lymphocytes were significantly increased ( $P \leq 0.014$ for each) (Figure $7, \mathrm{~A}$ and $\mathrm{B})$. At days 13,16 , and 21 , percentages of $\mathrm{CD}^{+} \mathrm{CD} 25^{+} \mathrm{T}$ cells rebounded ( $P \leq 0.038$ for each) after a decrease at days 8 and 10 ( $P \leq 0.0005$ for each), and throughout the chronic phase, the percentages of $\mathrm{CD} 4^{+} \mathrm{CD} 25^{+}$and $\mathrm{CD} 8^{+} \mathrm{CD} 25^{+} \mathrm{T}$ cells were significantly lower $(P \leq 0.026$ for each) relative to preinfection levels (Figure 7, A and B). A similar pattern was observed for the absolute counts of $\mathrm{CD} 4^{+} \mathrm{CD} 25^{+}$and $\mathrm{CD} 8^{+} \mathrm{CD} 25^{+} \mathrm{T}$

protein concentrations in plasma. Plasma TNF- $\alpha$ in all 6 SIVagminfected AGMs measured throughout the acute phase and at day 120 always remained under the detection limit of the assay $(\leq 15$ $\mathrm{pg} / \mathrm{ml}$ ) (data not shown). In contrast, we detected heightened levels of IL-10 in plasma during primary infection, and the increases were statistically significant at days $6,8,10$, and 13 ( $P \leq 0.002$ for each) (Figure $6 \mathrm{~B})$. In order to confirm that the early antiinflammatory cytokine environment was specific for SIVagm infection, we assayed IL-10 protein concentrations in 6 SIVmac251-infected rhesus macaques. They were significantly decreased from days 1 to 10 $(P \leq 0.019$ for each) and significantly increased only later on day $21(P=0.024)$ (Figure 6B). These data support the previous conclusion that an antiinflammatory environment is induced early in SIVagm infection compared with pathogenic SIVmac infection.

Transient IFN- $\gamma$ gene expression increases during primary SIVagm infection. IL-10 has been implicated in the suppression of Th1-type immune responses. We therefore followed the changes in the gene expression of a typical Th1-type cytokine, IFN- $\gamma$. We first compared IFN- $\gamma$ gene expression in the 3 tissue compartments of the 3 cells, the percentages of $\mathrm{CD} 4^{+} \mathrm{CD} 25^{+}$in $\mathrm{CD} 4^{+} \mathrm{T}$ cells, and the percentages of $\mathrm{CD}^{+} \mathrm{CD} 25^{+}$in $\mathrm{CD} 8^{+} \mathrm{T}$ cells (data not shown).

Immediate increases of TGF- $\beta 1$ and FoxP3 gene expressions during SIVagm. CD25 is not a specific marker of Tregs, as it is also upregulated upon conventional $\mathrm{T}$ cell activation. Recently, a transcription factor (FoxP3) has been described to be specifically expressed in humans by thymic and peripheral regulatory $\mathrm{CD}^{+}$and $\mathrm{CD}^{+}{ }^{+} \mathrm{CD} 25^{+} \mathrm{T}$ cells $(20,21)$. To determine whether the increased levels of $\mathrm{CD} 4^{+} \mathrm{CD} 25^{+}$and $\mathrm{CD}^{+} \mathrm{CD} 25^{+} \mathrm{T}$ cells observed in SIVagm-infected AGM were Tregs or conventional activated $\mathrm{T}$ cells, we quantified FoxP3 transcript levels in PBMCs of the same 6 animals. We found significantly increased FoxP3 levels at days 1 and 6 after infection (Figure 7C). Correlations between both numbers and percentages of $\mathrm{CD} 4{ }^{+} \mathrm{CD} 25^{+} \mathrm{T}$ cells and FoxP3 gene expression trended toward significance $(\rho=0.280, P=0.076$ and $\rho=0.278, P=0.079$, respectively). A statistically significant positive correlation was observed between FoxP3 gene expression and both the numbers $(\rho=0.548, P=0.0003)$ and percentages of CD ${ }^{+}$CD $25^{+}$T cells $(\rho=0.554, P=0.0002)$. 

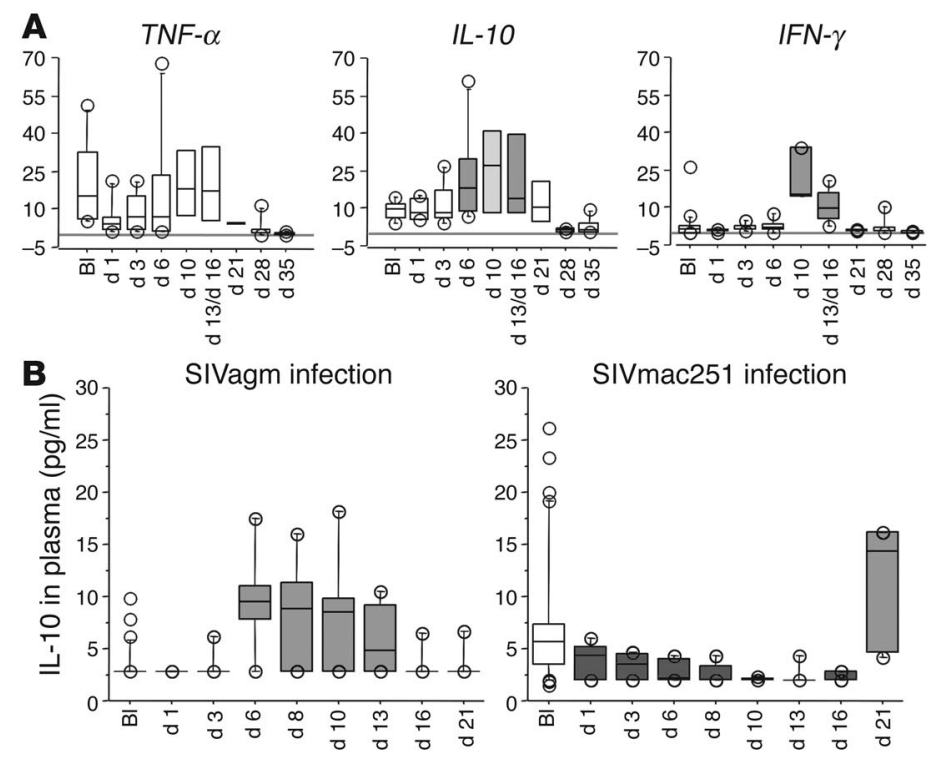

It has been previously found that FoxP3 gene expression and subsequent conversion of naive $\mathrm{CD} 4{ }^{+} \mathrm{CD} 25^{-} \mathrm{T}$ cells into $\mathrm{CD} 4^{+} \mathrm{CD} 25^{+}$ Tregs is induced by TGF- $\beta 1$ (22). Accordingly, we monitored $T G F-\beta 1$ gene expression in PBMCs during SIVagm infection. We observed statistically significant increased levels of TGF- $\beta 1$ (Figure 7D). The changes were observed very early at days 1,3 , and $13-16$ after infection $(P=0.0008, P=0.004$, and $P=0.028$, respectively) and there was a trend toward significance at day $6(P=0.079)$. The levels of FoxP3 and TGF- $\beta 1$ gene expression were highly correlated $(\rho=0.863, P<0.0001)$. Interestingly, immediate increases (day 1 after infection) were only observed for FoxP3 and TGF- $\beta 1$, whereas the expression of other cytokines, such as IL-10, was only increased several days later during primary infection. According to covariance analysis, TGF- $\beta 1$ gene expression had a significant effect after SIVagm infection on gene expressions of the other cytokines $(P=0.044$, multivariate analysis of covariance [MANCOVA] $)$, whereas $I L-10$ expression did not have a significant effect $(P=0.72$, MANCOVA). TGF- $\beta 1$ gene expression did not correlate with the numbers of CD $4^{+} \mathrm{CD} 25^{+} \mathrm{T}$ cells $(\rho=0.251, P=0.112)$ but did correlate with the percentages of $\mathrm{CD}^{+} \mathrm{CD} 25^{+} \mathrm{T}$ cells $(\rho=0.372, P=0.019)$ and, as had FoxP3, with both numbers $(\rho=0.557, P=0.0002)$ and percentages of $\mathrm{CD} 8{ }^{+} \mathrm{CD} 25^{+} \mathrm{T}$ cells $(\rho=0.554, P=0.0002)$.

To confirm the increases of TGF- $\beta 1$ gene expression at the protein level, we assayed TGF- $\beta 1$ in plasma during primary SIVagm infection. Our results clearly revealed a statistically significant increase in plasma TGF- $\beta 1$ at day 1 after infection $(P<0.0001)$ (Figure 7E). Then plasma concentrations returned to baseline levels at days 3 and 6 (except for 2 of the 6 animals at day 3 [SAB97008 and SAB98013] and at day 6 [SAB01015 and SAB00020], for which concentrations of plasma TGF- $\beta 1$ remained higher [2- to 3-fold] compared with their preinfection levels). Throughout days 10-21, concentrations of plasma TGF- $\beta 1$ were lower than preinfection baseline levels $(P<0.0001)$. Again, we found that TGF- $\beta 1$ concentrations in plasma were significantly correlated with the absolute numbers of $\mathrm{CD}^{+} \mathrm{CD} 25^{+} \mathrm{T}$ cells in blood $(\rho=0.375, P=0.033)$. This early rise of TGF- $\beta 1$ concentrations in plasma is consistent with our previous conclusion that an antiinflammatory cytokine environment is immediately induced after SIVagm infection.

\section{Figure 6}

Early pro- and antiinflammatory cytokine profiles in PBMCs and plasma. (A) $T N F-\alpha, I L-10$, and IFN- $\gamma$ gene expressions in PBMCs during primary SIVagm infection. Gene expressions were analyzed in 6 SIVagm-infected AGMs (SAB97008, SAB98013, SAB00020, SAB01013, SAB01015, and SAB02003) before and after infection, including very early time points (days 1 and 3 after infection). Values are expressed as $n$-fold increases relative to the calibrator. (B) Concentrations of IL-10 were measured in plasma collected from the same 6 SIVagm-infected AGMs and compared with 6 SIVmac251-infected rhesus macaques. Medium gray and dark gray boxes indicate statistically significant increases and decreases, respectively $(P<0.05$, Wilcoxon signed rank test). The light gray box indicates a trend toward significant increase (IL-10 gene expression at day $10, P<0.06$, Wilcoxon signed rank test).

In order to study whether or not the early TGF- $\beta 1$ increase is present during primary pathogenic SIVmac infection, we assayed TGF- $\beta 1$ in plasma from the 6 SIVmac251-infected rhesus macaques for which we had assayed IL-10 concentrations in plasma. We observed significant increases at days 1 , $3,6,10,13$, and 16 ( $P \leq 0.043$ for each) (Figure $7 F)$. Nevertheless, the increases at day 1 after SIVmac infection were significantly lower compared with those after SIVagm $(P=0.037$, Mann-Whitney $U$ test). Interestingly, the profiles were inversed after $V L$ peaked (days 10-21). Indeed, TGF- $\beta 1$ concentrations in infected macaques were significantly higher than those in infected AGMs at days 10 and $16(P=0.037$ and $P=0.025$, respectively, Mann-Whitney $U$ test). Therefore, the patterns were different in pathogenic and nonpathogenic infections, with an immediate strong increase in TGF- $\beta 1$ levels after primary SIVagm infection and a moderate persistent increase after primary SIVmac infection.

\section{Discussion}

The present study on nonpathogenic SIVagm infection adds significantly to our understanding of critical factors involved in AIDS protection. Several mechanisms have been proposed as driving progression toward AIDS in humans and macaques: (a) accelerated cell death, (b) impaired $\mathrm{T}$ cell generation, and (c) accelerated turnover leading to exhaustion. Our results show that during primary SIVagm infection in its natural host, a decline in peripheral $\mathrm{CD}^{+} \mathrm{T}$ cells to levels similar to those found in pathogenic SIVmac can occur. The $\mathrm{CD}^{+} \mathrm{T}$ cell decline was slightly greater than that of $\mathrm{CD}^{+} \mathrm{T}$ cells and may thus partially result from virus-induced cell death. SIVagm has indeed been shown to be as cytopathic as HIV-1 or SIVmac in vitro (23). However, since $\mathrm{CD}^{+} \mathrm{T}$ cells also decrease, albeit to a lesser extent than $\mathrm{CD}^{+} \mathrm{T}$ cells, an additional reason for loss of $\mathrm{T}$ cells in the periphery could be bystander cell death and/or migration to LN. Bystander cell death of $\mathrm{CD}^{+} \mathrm{T}$ cells has been demonstrated in several natural hosts, including AGMs (13). Although we did not see any changes in the $\mathrm{CD} 4^{+} / \mathrm{CD}^{+} \mathrm{T}$ cell ratio in $\mathrm{LN}$, a slight, equal increase in both $\mathrm{CD} 4^{+}$and $\mathrm{CD} 8^{+} \mathrm{T}$ cells cannot be excluded. If such an increase did occur, it must be moderate in comparison with that in SIVmac-infected macaques, since no lymphadenopathy has been evidenced in LN of SIVagm-infected AGMs (9). The detection of increased percentages of activated $\mathrm{CD}^{+} \mathrm{T}$ cells during primary infection in blood but not in $\mathrm{LN}$ of AGMs might be explained by a release of activated cells in the circulation in AGMs. In contrast, the higher and more persistent levels of activated $\mathrm{T}$ cells in $\mathrm{LN}$ in macaques suggests a sequestration 
A
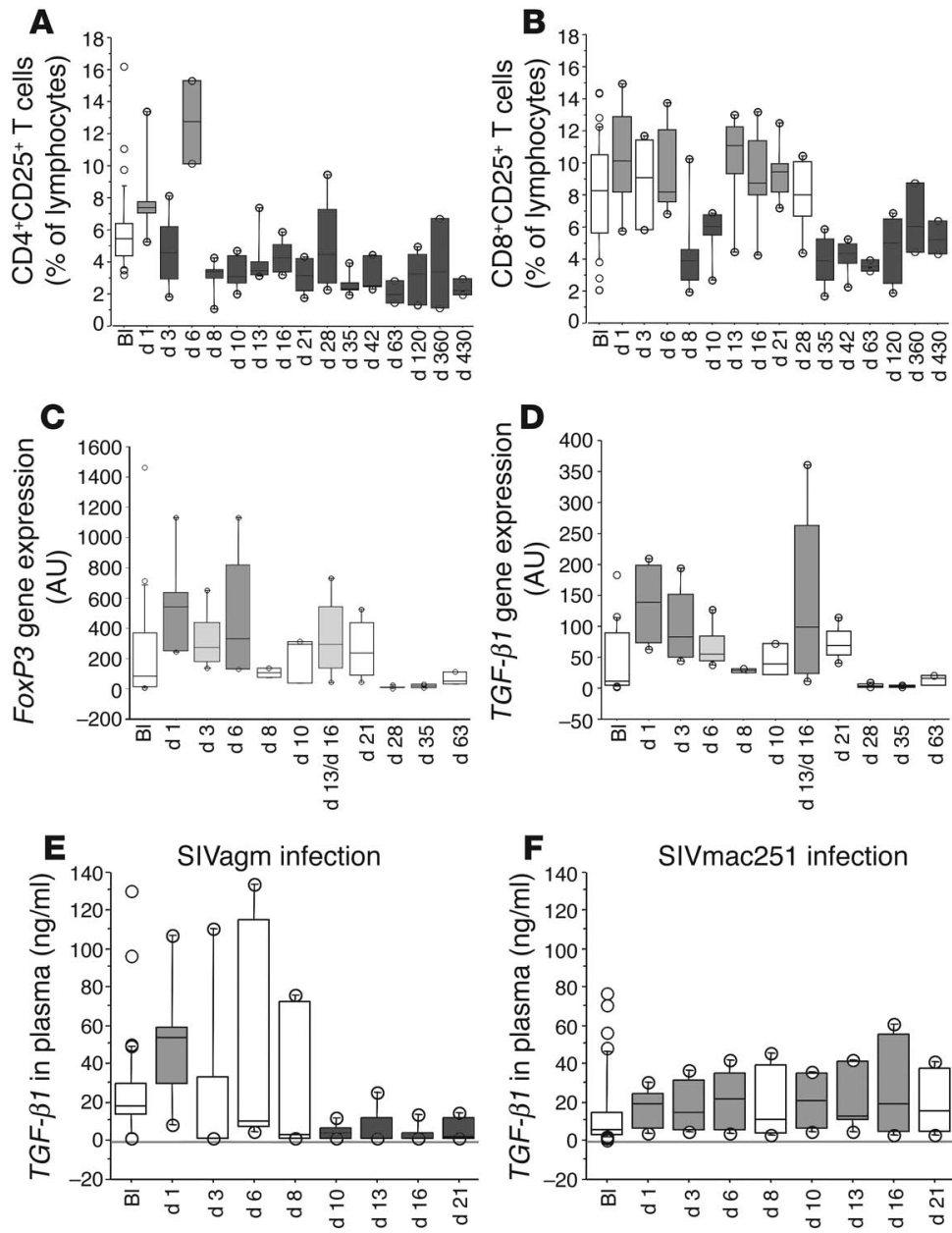

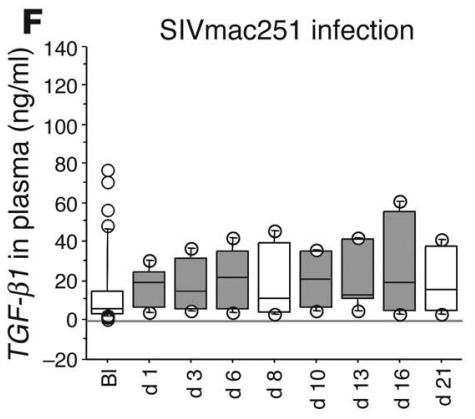

\section{Figure 7}

Assessment of CD25+ $T$ cell percentages and of the expression levels of FoxP3 and TGF- $\beta 1$. (A) CD4+CD25+ $T$ cell percentages within lymphocytes of 6 SIVagm-infected AGMs (SAB97008, SAB98013, SAB00020, SAB01013, SAB01015, and SAB02003). (B) CD8 ${ }^{+} \mathrm{CD} 25^{+} \mathrm{T}$ cell percentages within lymphocytes of these AGMs. (C) FoxP3 gene expression in PBMCs of these AGMs. (D) TGF- $\beta 1$ gene expression in PBMCs of these AGMs. (E) Concentrations of TGF- $\beta 1$ in plasma of these 6 AGMs. (F) Concentrations of TGF- $\beta 1$ in plasma of 6 SIVmac251-infected rhesus macaques. See also legend to Figure 6 for medium gray and dark gray boxes.

infection, as observed in some LTNPs (26). Although, Th1 responses are protective, they may be harmful if they are associated with strong, generalized immune activation $(12,27)$. An et al. indeed reported that a TNF- $\alpha-$ inducible promoter variant of IFN- $\gamma(-179 T)$ accelerates loss of $\mathrm{CD}^{+} \mathrm{T}$ cells in humans (28). In clinical trials with IFN- $\gamma$, both acceleration of AIDS progression and decline of CD4 cell counts have been reported (29). Recently, it has been shown that the long-term challenge outcome in vaccinated macaques was correlated with the relative balance between SIV-specific IFN- $\gamma$ T cell responses and nonspecific IFN- $\boldsymbol{\gamma}$-driven inflammation, the latter being seen in animals in which viral replication cannot be controlled (30).

We found that an antiinflammatory environment is established early during SIVagm infection. Our data on $\beta$ chemokine expression are in contrast to those on HIV-1 infection in humans or SIVmac-infected macaques, where significant increases of RANTES, MIP-1 $\alpha, M I P-1 \beta$, and CCR5 gene expressions have been observed in PBMCs, LN, and BALCs $(17,18)$. Recently, it has been reported that high $\beta$ chemokine expression levels in lym-

of activated cells in LN during pathogenic HIV-1/SIVmac infections. This hypothesis is consistent with the lack of infiltration of $\mathrm{CD}^{+}$cells in germinal centers of LN of infected AGMs (9).

The lack of chronic hyperactivation seems indeed to be a common feature of nonpathogenic SIV infections in natural hosts (8-11, 13, 24). In HIV infection, evidence has been accumulating to support the concept that the degree of activation is the best predictor of disease progression, whereas VL is only indirectly associated with progression $(1,2,4) . \mathrm{CD}^{+} \mathrm{T}$ cell activation at early stages in HIV infection is determinant of the rate at which $\mathrm{CD}^{+} \mathrm{T}$ cells are lost over time (3). Thus, it is critical to identify the mechanism(s) that protect against the harmful hyperactivation of the immune system. It has been proposed that protection in AGMs could be due to tolerance against a SIVagm protein (25). Our study reveals a state of increased $\mathrm{T}$ cell activation in peripheral blood subsequent to SIVagm infection, which thus clearly demonstrates that AGMs respond to SIVagm infection and that protection is not due to a central tolerance mechanism. However, our data on $I F N-\gamma$ gene expression suggest a low Th1 response. We found no increase in LNLCs and BALCs and increases in PBMCs only between days 5 and 16 after infection. This is consistent with the decreased IFN- $\gamma$ production in $\mathrm{CD}^{+} \mathrm{T}$ cells and low CTL frequencies reported in chronically SIVsm-infected SMs $(11,12)$. It is beneficial to maintain a Th1-type cytokine secretion pattern when it is associated with efficient CTL responses during chronic HIV-1 phoid tissues of SIVmac-infected macaques contribute to nonspecific inflammation and immune activation as well as to high VL in lymphoid tissues (31). RANTES is chemotactic mainly for monocytes and CD4 ${ }^{+}$memory T cells, whereas MIP- $1 \alpha$ and MIP-1 $\beta$ have been shown to attract $\mathrm{CD}^{+} \mathrm{T}$ cells (32). Therefore, the lack of $M I P-1 \alpha$ and MIP-1 $\beta$ gene overexpression in SIVagm infection might be associated with the lack of CD8 $8^{+}$lymphocytosis and $\mathrm{CD}^{+}$infiltration in $\mathrm{LN}$ germinal centers (9).

SIVagm-infected AGMs also exhibited no signs of significant $T N F-\alpha$ increases. This contrasts with $T N F-\alpha$ increases detected in acutely SIVmac-infected macaques and in HIV-infected humans $(14,33,34)$. We previously showed, for instance, that SIVmacinfected cynomolgus macaques produce up to $1,500 \mathrm{pg}$ TNF- $\alpha$ / $\mathrm{ml}$ of serum during primary infection (35). The lower TNF- $\alpha$ expression during SIVagm infection was not due to a constitutive impairment, since we detected TNF- $\alpha$ (up to $100 \mathrm{pg} / \mathrm{ml}$ ) after ConA stimulation of AGM mononuclear cells (data not shown). Rather, as shown here, AGMs exhibit a significant increase of IL-10 during primary infection. Although increased $I L-10$ expression levels have been associated with disease progression during later stages of HIV-1 infection (36), a higher stimulus-induced IL-10 mRNA expression, together with a lower constitutive TNF- $\alpha$ mRNA expression - similar to what we observed in AGMs - were indeed associated with slower disease progression in HIV-1-infected children (37). Moreover, a mutation in the $I L-10$ promoter 
region, resulting in constitutively reduced $I L-10$ gene expression, is associated with a more rapid progression toward AIDS in HIV-1infected patients (38). One explanation for the discrepancy in the findings on the role of IL-10 might thus be related to the different disease stages studied. Here our data show that during acute SIVmac251 infection in rhesus macaques, IL-10 secretion in plasma was indeed significantly increased, but this occurred later than in nonpathogenic SIVagm infection. Previous studies on SIVmacinfected macaques showed enhanced levels of $I L-10$ gene expression in PBMCs in a few animals during primary SIVmac infection, but this expression was generally accompanied by strong TNF- $\alpha$ gene expressions $(14,19)$. Together, our data reveal early induction of an antiinflammatory environment in response to SIVagm infection in AGMs. This may be what determines the subsequent low, generalized immune activation levels.

This antiinflammatory environment might be induced in AGMs by TGF- $\beta 1$, whose expression is upregulated as early as 24 hours after infection to levels significantly higher than those in macaques, which show greater levels only starting at day 10 . Omer et al. observed that in Plasmodium yoelii-infected mice, a burst of TGF- $\beta 1$ within 24 hours inhibits IFN- $\gamma$ and TNF- $\alpha$ responses, whereas increases of TGF- $\beta 14$ days later do not (39). Moreover, the environment in which TGF- $\beta 1$ is produced starting from the second week after infection is also different between pathogenic and nonpathogenic SIV infections, since in macaques it is concomitant with a proinflammatory cytokine balance $(14,17-19$, $33,35)$. The response by TGF- $\beta 1$ that is weaker early and stronger thereafter in macaques may reflect an inability to prevent a fatal overwhelmed immune response and a continuous need for tissue repair mechanisms, as conferred by TGF- $\beta 1$ (40), due to an inability to resorb virus-driven inflammation. In Plasmodium yoelii-infected mice, the major producers of TGF- $\beta 1$ are adherent cells, $\mathrm{CD}^{+}$and $\mathrm{CD} 25^{+}$cells (39). In AGMs, we found that the most statistically significant correlation of TGF- $\beta 1$ (transcript and plasma levels) was with $\mathrm{CD} 8{ }^{+} \mathrm{CD} 25^{+} \mathrm{T}$ cells. It remains to be determined which cells express TGF- $\beta 1$ during primary nonpathogenic SIVagm infection and pathogenic SIVmac infection, but $\mathrm{CD}^{+} \mathrm{CD} 25^{+} \mathrm{T}$ cells could be one of the major sources of TGF- $\beta 1$ during primary SIVagm infection.

TGF- $\beta 1$ might be responsible for the induction of FoxP3, which correlates with the increases of $\mathrm{CD}_{25} 5^{+}$cells, in particular $\mathrm{CD}^{+} \mathrm{CD} 25^{+} \mathrm{T}$ cells. Although the presence of Tregs in AGMs needs to be confirmed by functional studies, the hypothesis that they are induced is strongly supported by our findings. So far, it is unclear whether they would correspond to natural (Trn) or to peripherally induced ( $\operatorname{Tr} 1, \mathrm{TH} 3)$ Tregs or both. One cannot exclude the possibility that a first expansion of FoxP3 ${ }^{+}$Trn might enhance the induction of peripheral IL-10-producing FoxP3Tregs, eventually associated with the repeated Ag stimulations in the absence of proinflammatory signals (41). Further studies are needed to determine to which subset of $\mathrm{CD}^{+}$and/or $\mathrm{CD} 4^{+}$Tregs the cells might belong.

Such an induction of Tregs could explain the downregulation of $\mathrm{T}$ cell activation in AGMs. The presence of $\mathrm{CD}^{+}$and $\mathrm{CD}^{+}$Tregs has been described ex vivo during the chronic phase in HIV-1infected patients (42-46). Some of these studies proposed that an impaired generation of $\mathrm{CD} 4{ }^{+} \mathrm{CD} 25^{+}$Tregs might be at the origin of the uncontrolled $\mathrm{T}$ cell hyperactivation $(44,45)$, whereas others proposed that Tregs could be at the origin of immunosuppression in HIV-1 infection $(42,46)$. Thus, it is not clear so far whether they play a protective role against $\mathrm{T}$ cell hyperactivation or rather contribute to immunosuppression in HIV infection. Our data are in favor of a protective role of Tregs. We and others have previously shown that primary SIVmac infection is associated with increases in $\mathrm{CD}^{+} \mathrm{CD} 25^{+} \mathrm{T}$ cells in blood between days 7 and 21 after infection but with unchanged $\mathrm{CD} 8{ }^{+} \mathrm{CD} 25^{+} \mathrm{T}$ cell levels $(47,48)$. One can hypothesize that SIVmac-infected macaques exhibit chronic abnormal $\mathrm{T}$ cell activation due to a delayed expansion and/or a lack of $\mathrm{CD}^{+}$Treg induction.

In summary, our study describes, for the first time to our knowledge, the balance between the expression of pro- versus antiinflammatory cytokines within the first days after infection during nonprogressive SIV infection, and suggests a mechanism for avoiding harmful generalized immune activation after lentiviral infection. Our data reveal an immediate antiinflammatory balance in AGMs in response to SIVagm infection. This balance might be primed by TGF- $\beta 1$, which is induced early and strongly and which might lead to the very early increase (starting from day 1 after infection) of FoxP3 expression. The potential rapidly induced $\mathrm{CD}^{+}$and $\mathrm{CD}^{+}$Tregs might temper the proinflammatory cytokine cascade and protect against chronic hyperactivation. These new insights into the early cytokine balance and potential protective role of Tregs are crucial for the research on novel immune intervention strategies for the prevention and treatment of AIDS.

\section{Methods}

Viral stocks. The generation and titration of the SIVagm.sab92018 virus stock has been described elsewhere (9). The cell-free SIVmac251 stock was kindly provided by A.M. Aubertin (Université Louis Pasteur, Strasbourg, France). We used AGMs inoculated with SIV-negative plasma as mock-

\section{Table 1}

Primers and probes used for cellular gene expression analysis in AGMs

\begin{tabular}{|c|c|c|c|}
\hline Marker & FW primer [5'-3'orientation] & REV primer [5'-3'orientation] & TaqMan probe [5'FAM-3'TAMRA] \\
\hline CCR1 & GTCATGTTTTTGAGCCTCTTGTATTG & GGTGAATGAGAGGGCCATTTT & TTCCAACCACGCCAATGACAAATACCA \\
\hline CCR5 & CAAAGGTGACTGTCCTGGCTTT & TTCTCTGGAATCTTCTTCATCATCC & AACACAGCATGGACGATAGCCAGGTACC \\
\hline$I L-10$ & TTGCTGGAGGACTTTAAGGGTTAC & TCAGCTTGGGGCATAACC & TTGCCAAGCCTTGTCTGAGATGATCCA \\
\hline$T N F-\alpha$ & ATGGCAGAGAGGAGGTTGACC & TACTCCCAGGTCCTCTTCA & CAGCCGCATCGCCGTCTCCT \\
\hline IFN- $\gamma$ & CCAAATTGTCTCCTTTTACTTCAAACTT & CATCCCGTTTCTTTTTGTTGCTAT & CCAAAAGAGTGTGGAGACCATCAAGGAAGA \\
\hline$M I P-1 \alpha$ & CCGGCAGATTCCACAGAATTT & TCAGGCACTCAGCTCCAGGT & CAAGCCCGGTGTCATTTCCTAACCAA \\
\hline 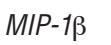 & ACTGCGAGGAGTCCTGA & ATGACCTGGAACTGAACTGAGCT & TGACCTTCCCTGAAGACTTCCTGTCTCTGA \\
\hline RANTES & CGCTCCTGCATCTGCCTC & TTGGAGCACTTGCCACTGG & TTTGCCTACATTGCCCGCCCACT \\
\hline
\end{tabular}

FW, forward; REV, reverse. 
infected controls. This plasma was collected from the same monkey from which samples were used to prepare the SIVagm stock. The negative control plasma for macaques was obtained from an unrelated donor macaque.

Animals and infections. AGMs used in these studies belonging to the Chlorocebus sabaens species were housed at the Pasteur Institute facility in Dakar, Senegal. Cynomolgus macaques (MAC141A, MACN894, MACQ172, and MACQ205) were housed at the Centre International de Recherche Medicale in Franceville, Gabon. Chinese rhesus macaques (MAC1058, MAC1073, MAC1076, MAC1085, MAC1094, and MAC1099) were housed at the Pasteur Institute facility in Ile-de-France, France. All animals were housed according to national guidelines and institutional policies. Animal care and use were approved by the Committee for Ethics and Animal Experimentation at the International School of Science and Veterinary Medicine in Dakar, Senegal. For infections, 100 and 300 $\mathrm{TCID}_{50}$ (where $\mathrm{TCID}_{50}$ is the tissue culture infective dose that affects $50 \%$ of inoculated cultures) SIVagm.sab92018 were intravenously inoculated into 3 (SAB98007, SAB98008, and SAB98011) and 6 (SAB97008, SAB98013, SAB00020, SAB01013, SAB01015 and SAB02003) sabaeus monkeys (a sub-species of AGMs), respectively. The 3 cynomolgus macaques were intravenously inoculated with $100 \mathrm{TCID}_{50}$ of SIVmac251. The 6 rhesus macaques were intravenously inoculated with 10 AID $_{50}$ (where $\mathrm{AID}_{50}$ is the animal infective dose that affects $50 \%$ of inoculated cultures) of SIVmac251. Four control animals (2 sabaeus and 2 cynomolgus macaques) were intravenously inoculated with the corresponding volume $(0.03-1 \mathrm{ml})$ of species-specific SIV-negative plasma. Although, we cannot exclude the possibility that differences between SIVagm.sab92018 and SIVmac251 infections described here are virus dependent, we used models in which monkeys were infected with their species-specific SIVs.

Specimen collection. For every monkey included in the protocol, $5 \mathrm{ml}$ of whole blood was collected in EDTA K2 tubes. PBMCs were isolated by Ficoll-Hypaque (Sigma-Aldrich) gradient centrifugation, after which plasma was recovered and clarified from cellular debris and residual platelets by additional centrifugation at $3,000 \mathrm{~g}$ for 5 minutes.

Excisional axillar and inguinal LN biopsies were performed, and tissues were mechanically dissociated. LNLCs were separated from the remaining tissue by passage through a $100-\mathrm{mm}$ nylon mesh. A total of $2 \times 10^{6}$ fresh cells were used for flow cytometry.

BAL was carried out after local anesthesia with lignocaine (Xylovet; Sanofi-Synthelabo) (18).

Flow cytometric analyses. The following monoclonal antibody clones were used: CD3-FITC (FN18; BioSource International), CD4-PE (Leu3; BD), CD8-PE (Leu2a; BD), CD16-PE (3G8; BD Biosciences - Pharmingen), CD25-PE (1HT44H3; Immunotech/Beckman Coulter Inc.), CD28-FITC (L293; BD), and HLA-DR-FITC (L243; BD), as well as isotype controls matching the IgG subclass. AGMs often demonstrate a high frequency of $\mathrm{CD} 4^{+} \mathrm{T}$ cells coexpressing CD8 $(6,49)$. Double-positive $\mathrm{CD} 4^{+} / \mathrm{CD}^{+} \mathrm{T}$ cells express the $\alpha$ chain at low density and not the $\beta$ chain, whereas the majority of $\mathrm{CD} 4-/ \mathrm{CD} 8 \alpha^{\text {bright }}$ cells coexpress the $\beta$ chain (49). CD $8 \alpha^{\text {bright }} / \mathrm{CD} 4^{-}$cells were defined as $\mathrm{CD}^{+} \mathrm{T}$ cells and the $\mathrm{CD}^{+} / \mathrm{CD} 8 \alpha^{\text {low }}$ or $\mathrm{CD}^{+} / \mathrm{CD} 8^{-} \mathrm{T}$ cells as $\mathrm{CD} 4^{+} \mathrm{T}$ cells. Absolute numbers of lymphocyte subsets in blood were calculated using routine diagnostic counts.

RNA extraction from tissues. PBMCs, LN lymphocytes, and BALCs were lysed in RLT-RNA lysis buffer (QIAGEN) and then were run over a QIAshredder column (QIAGEN) to ensure homogeneous lysis. Thereafter, we extracted total RNA using the RNeasy RNA extraction Mini Kit (QIAGEN) and by adding an RNAse-free DNAseI (QIAGEN) treatment on the column.

Quantification of viral RNA copy numbers in plasma by real-time PCR. The RNA copy numbers of SIVagm.sab and SIVmac were quantified by realtime PCR. The RT-PCR assay specific for SIVagm.sab has been described elsewhere (9). Quantification of SIVmac RNA in real time was carried out using a protocol initially described by Hofmann-Lehmann et al. (15). The standard RNA was obtained from the pGEM-5Zf+ GAG plasmid (provided by A.-M. Aubertin, Université Pasteur, Strasbourg, France).

Quantification of cellular gene expression by real-time PCR. Total RNA primed with random hexamers was submitted to an RT step and carried out with the TaqMan Reverse Transcription Reagents kit (Applied Biosystems). The primers and TaqMan probes used for the quantification are summarized in Table 1. For the quantitation of FoxP3 and TGF- $\beta 1$ gene expression, we used commercially available kits (TaqMan Assay-onDemand; Applied Biosystems).

To normalize for RNA input, the sample content of $18 \operatorname{SrRNA}$ was quantified with the TaqMan assay reagents for human 18SrRNA (Applied Biosystems). We determined the expression of a given gene using the comparative $\left(\Delta \Delta \mathrm{C}_{\mathrm{T}}\right)$ method (User Bulletin 2, ABI PRISM 7700 Sequence Detection System; http://docs.appliedbiosystems.com/pebiodocs/04303859. pdf). The expression levels of each gene normalized for $18 S r R N A$ are expressed as $n$-fold difference relative to the calibrator corresponding to total cellular RNA from an unrelated sample.

Quantification of cytokines in plasma. We measured levels of TNF- $\alpha$, IL-10, and TGF- $\beta 1$ in plasma using the following ELISA kits: Cytoscreen monkey TNF- $\alpha$ (Biosource International), IL-10 monkey (U-CyTech Biosciences), and TGF- $\beta 1$ Multispecies Cytoscreen (Biosource International), respectively. Detection limits were $15,2.5$, and $15.6 \mathrm{pg} / \mathrm{ml}$ for TNF- $\alpha$, IL-10, and TGF- $\beta 1$, respectively. To verify that the anti-macaque TNF- $\alpha$ and IL-10 antibodies cross-reacted with AGM cytokines, AGM PBMCs were stimulated in vitro, and cytokines were measured in the supernatants (data not shown).

Statistical analyses. We performed all statistical analyses using Statview (Abacus Concept). $P<0.05$ was considered statistically significant. We used the Wilcoxon signed rank test to evaluate whether there was a statistically significant difference in the level of one given marker at a given time point following inoculation when compared to the preinoculation baseline. Preinoculation baselines consisted of 4 preinfection values per animal for the gene expression analysis and 7 total preinfection values for the study of plasma cytokines and of CD25+ $\mathrm{T}$ cell frequency and counts. The Spearman rank test was used to assess the correlation between 2 continuous variables. All analyzed markers were previously normalized in $\log _{10}$. We performed MANCOVA to study the simultaneous effect of the nominal factor before infection versus after SIVagm infection and (a) TGF- $\beta 1$ gene expression as a covariate, on TNF- $\alpha, I F N-\gamma$, and $I L-10$ gene expressions and (b) $I L-10$ gene expression as a covariate, on TNF- $\alpha, I F N-\gamma$, and TGF- $\beta 1$ gene expressions. We used the Mann-Whitney $U$ test to compare the cytokine concentrations in plasma between SIVagm and SIVmac infections.

\section{Acknowledgments}

We thank L. An for excellent technical help. We are indebted to J.M. McCune, L. Rogge, and D. Scott-Algara for critical reading of this manuscript. C. Kornfeld received a fellowship from SIDACTION; M.J.-Y. Ploquin, a scholarship from Le Ministère de l'Education Nationale, de la Recherche et de la Technologie; and L. Mortara and J.-F. Desoutter, fellowships from the French Agency for AIDS Research (ANRS). The study was supported by grants from the ANRS.

Received for publication August 11,2004, and accepted in revised form January 18, 2005.

Address correspondence to: Michaela Müller-Trutwin, Département de Virologie, Unité de Biologie des Rétrovirus, 25-28 rue du Docteur Roux, F-75724 Paris Cedex 15, France. Phone: 33-1-40-6139-69; Fax: 33-1-45-68-89-57; E-mail: mmuller@pasteur.fr. 
C. Kornfeld and M.J.-Y. Ploquin contributed equally to this work.

I. Pandrea and C. Apetrei's present address is: Tulane National Primate Research Center, Tulane University Health Sciences Center, Covington, Louisiana, USA.

1. Sousa, A.E., et al. 2002. CD4 T cell depletion is linked directly to immune activation in the pathogenesis of HIV-1 and HIV-2 but only indirectly to the viral load. J. Immunol. 169:3400-3406.

2. Hazenberg, M.D., et al. 2003. Persistent immune activation in HIV-1 infection is associated with progression to AIDS. AIDS. 17:1881-1888.

3. Deeks, S.G., et al. 2004. Immune activation setpoint during early HIV infection predicts subsequent CD4+ Tt cell changes independent of viral load. Blood. 104:942-947.

4. van Asten, L., et al. 2004. Pre-seroconversion immune status predicts the rate of CD4 $\mathrm{T}$ cell decline following HIV infection. AIDS. 18:1885-1893.

5. Apetrei, C., Robertson, D.L., and Marx, P.A. 2004. The history of SIVs and AIDS: epidemiology, phylogeny and biology of isolates from naturally SIV infected non-human primates (NHP) in Africa. Front. Biosci. 9:225-254.

6. Muller, M.C., and Barre-Sinoussi, F. 2003. SIVagm: genetic and biological features associated with replication. Front. Biosci. 8:d1170-d1185.

7. Gueye, A., et al. 2004. Viral load in tissues during the early and chronic phase of non-pathogenic SIVagm infection. J. Med. Primatol. 33:83-97.

8. Chakrabarti, L. 2004. The paradox of SIV infection in sooty mangabeys: active viral replication without disease progression. Front. Biosci. 9:521-539.

9. Diop, O.M., et al. 2000. High levels of viral replication during primary SIVagm infection are rapidly and strongly controlled in African Green Monkeys. J. Virol. 74:7538-7547.

10. Pandrea, I., et al. 2003. High levels of SIVmnd-1 replication in chronically infected Mandrillus sphinx. Virology. 317:119-127.

11. Silvestri, G., et al. Nonpathogenic SIV infection of sooty mangabeys is characterized by limited bystander immunopathology despite chronic highlevel viremia. Immunity. 18:441-452.

12. Ansari, A.A., et al. 2003. Lessons learnt from studies of the immune characterization of naturally SIV infected sooty mangabeys. Front. Biosci. 8:s1030-s1050.

13. Estaquier, J., et al. 1994. Programmed cell death and AIDS: significance of T-cell apoptosis in pathogenic and nonpathogenic primate lentiviral infections. Proc. Natl. Acad. Sci. U. S. A. 91:9431-9435.

14. Benveniste, et al. 1996. Comparative interleukin (IL-2)/interferon IFN-gamma and IL-4/IL-10 responses during acute infection of macaques inoculated with attenuated nef-truncated or pathogenic SIVmac251 virus. Proc. Natl. Acad. Sci. U. S. A. 93:3658-3663.

15. Hofmann-Lehmann, R., et al. 2000. Sensitive and robust one-tube real-time reverse transcriptasepolymerase chain reaction to quantify SIV RNA load: comparison of one- versus two-enzyme systems. AIDS Res. Hum. Retroviruses. 16:1247-1257.

16. Lebel-Binay, S., et al. 1999. The decline of $\mathrm{CD} 8+\mathrm{CD} 28+$ peripheral lymphocytes is correlated with the evolution of AIDS in macaques infected with SIVmac251. AIDS. 13:136-137.

17. Cheret, A., et al. 1999. RANTES, IFN-gamma, CCR1, and CCR5 mRNA expression in peripheral blood, lymph node, and bronchoalveolar lavage mononuclear cells during primary SIV infection of macaques. Virology. 255:285-293.

V. Poaty-Mavoungou's present address is: Institute for Tropical Medicine, Department of Parasitology, University of Tübingen, Tübingen, Germany.

L. Mortara's present address is: Department of Clinical and Biological Sciences, School of Medicine, University of Insubria, Varese, Italy.

18. Caufour, P., et al. 1999. Secretion of beta-chemokines by bronchoalveolar lavage cells during primary infection of macaques inoculated with attenuated nef-deleted or pathogenic SIV strain mac251. J. Gen. Virol. 80:767-776.

19. Cheret, A., et al. 1996. Cytokine mRNA expression in mononuclear cells from different tissues during acute SIVmac251 infection of macaques. AIDS Res. Hum. Retroviruses. 12:1263-1272.

20. Walker, M.R., et al. 2003. Induction of FoxP3 and acquisition of $\mathrm{T}$ regulatory activity by stimulated human $\mathrm{CD}^{+} \mathrm{CD} 25^{-} \mathrm{T}$ cells. J. Clin. Invest. 112:1437-1443. doi:10.1172/JCI200319441.

21. Cosmi, L., et al. 2003. Human CD8+CD25+ thymocytes share phenotypic and functional features with CD4+CD25+ regulatory thymocytes. Blood. 102:4107-4114.

22. Chen, W., et al. 2003. Conversion of peripheral CD4+CD25- naive T cells to CD4+CD25+ regulatory $\mathrm{T}$ cells by TGF-beta induction of transcription factor Foxp3. J. Exp. Med. 198:1875-1886.

23. Murayama, Y., et al. 1997. CD4 and CD8 expressions in African green monkey helper $\mathrm{T}$ lymphocytes: implication for resistance to SIV infection. Int. Immunol. 9:843-851.

24. Onanga, R., et al. 2002. High levels of viral replication contrast with only transient changes in CD4+ and CD8+ cell numbers during the early phase of experimental infection with SIVmnd-1 in Mandrillus sphinx. J. Virol. 76:10256-10263.

25. Norley, S., and Kurth, R. 2004. The role of the immune response during SIVagm infection of the African green monkey natural host. Front. Biosci. 9:550-564.

26. Migueles, S.A., et al. 2002. HIV-specific CD8+ T cell proliferation is coupled to perforin expression and is maintained in nonprogressors. Nat. Immunol. 3:1061-1068.

27. Fauci, A.S. 1993. Multifactorial nature of human immunodeficiency virus disease: implications for therapy. Science. 262:1011-1018.

28. An, P., et al. 2003. A tumor necrosis factor-alphainducible promoter variant of interferon-gamma accelerates CD4+ T cell depletion in HIV-1-infected individuals. J. Infect. Dis. 188:228-231.

29. Poli, G., Biswas, P., and Fauci, A.S. 1994. Interferons in the pathogenesis and treatment of HIV infection. Antiviral Res. 24:221-233.

30. Abel, K., et al. 2004. Gamma interferon-mediated inflammation is associated with lack of protection from intravaginal SIVmac239 challenge in SHIV89.6-immunized rhesus macaques. J. Virol. 78:841-854.

31. LaFranco-Scheuch, L., et al. 2004. High betachemokine expression levels in lymphoid tissues of SHIV89.6-vaccinated rhesus macaques are associated with uncontrolled replication of SIV challenge inoculum. J. Virol. 78:6399-6408.

32. Taub, D.D., et al. 1993. Preferential migration of activated $\mathrm{CD} 4+$ and $\mathrm{CD} 8+\mathrm{T}$ cells in response to MIP-1 alpha and MIP-1 beta. Science. 260:355-358.

33. Benveniste, O., et al. 1996. Interleukin 1 beta, interleukin 6 , tumor necrosis factor alpha, and interleukin 10 responses in peripheral blood mononuclear cells of cynomolgus macaques during acute infection with SIVmac251. AIDS Res. Hum. Retroviruses.
12:241-250.

34. Graziosi, C., et al. 1996. Kinetics of cytokine expression during primary HIV type 1 infection. Proc. Natl. Acad. Sci. U. S. A. 93:4386-4391.

35. Clayette, P., et al. 1995. Tumor necrosis factoralpha in serum of macaques during SIVmac251 acute infection. J. Med. Primatol. 24:94-100.

36. Clerici, M., et al. 1994. Role of interleukin-10 in T helper cell dysfunction in asymptomatic individuals infected with the human immunodeficiency virus. J. Clin. Invest. 93:768-775.

37. Than, S., et al. 1997. Cytokine pattern in relation to disease progression in human immunodeficiency virus-infected children. J. Infect. Dis. 175:47-56.

38. Shin, H.D., et al. 2000. Genetic restriction of HIV-1 pathogenesis to AIDS by promoter alleles of IL10. Proc. Natl. Acad. Sci. U. S. A. 97:14467-14472.

39. Omer, F.M., de Souza, J.B., and Riley, E.M. 2003. Differential induction of TGF-beta regulates proinflammatory cytokine production and determines the outcome of lethal and nonlethal Plasmodium yoelii infections. J. Immunol. 171:5430-5436.

40. Schmidt-Weber, C.B., and Blaser, K. 2004. Regulation and role of transforming growth factor-beta in immune tolerance induction and inflammation. Curr. Opin. Immunol. 16:709-716.

41. O'Garra, A., Vieira, P.L., Vieira, P., and Goldfeld, A.E. 2004. IL-10-producing and naturally occurring $\mathrm{CD}^{+}$Tregs: limiting collateral damage. J. Clin. Invest. 114:1372-1378. doi:10.1172/JCI200423215.

42. Aandahl, E.M., et al. 2004. Human CD4+ CD25+ regulatory $\mathrm{T}$ cells control $\mathrm{T}$-cell responses to human immunodeficiency virus and cytomegalovirus antigens. J. Virol. 78:2454-2459.

43. Garba, M.L., et al. 2002. HIV antigens can induce TGF-beta(1)-producing immunoregulatory CD8+ T cells. J. Immunol. 168:2247-2254.

44. Oswald-Richter, K., et al. 2004. HIV infection of naturally occurring and genetically reprogrammed human regulatory T-cells. PLoS Biol. 2:0955-0966.

45. Kinter, A.L., et al. 2004. CD25+CD4 regulatory T cells from the peripheral blood of asymptomatic $\mathrm{HIV}$-infected individuals regulate $\mathrm{CD} 4+$ and CD8+ HIV-specific T cell immune responses in vitro and are associated with favorable clinical markers of disease status. J. Exp. Med. 200:331-343.

46. Weiss, L., et al. 2004. Human immunodeficiency virus-driven expansion of CD4+CD25+ regulatory $\mathrm{T}$ cells, which suppress HIV-specific CD4 T-cell responses in HIV-infected patients. Blood. 104:3249-3256.

47. Estaquier, J., et al. 2000. Early changes in peripheral blood $\mathrm{T}$ cells during primary infection of rhesus macaques with a pathogenic SIV. J. Med. Primatol. 29:127-135.

48. Giavedoni, L.D., et al. 2000. Cytokine expression, natural killer cell activation, and phenotypic changes in lymphoid cells from rhesus macaques during acute infection with pathogenic SIV.J. Virol. 74:1648-1657.

49. Holznagel, E., Norley, S., Holzammer, S., Coulibaly, C., and Kurth, R. 2002. Immunological changes in simian immunodeficiency virus (SIVagm)-infected African green monkeys (AGM): expanded cytotoxic T lymphocyte, natural killer and B cells subsets in the natural host of SIVagm. J. Gen. Virol. 83:631-640. 